\title{
1 Analysis of negative capacitance and self-heating in organic semiconductor devices
}

\author{
Evelyne Knapp ${ }^{1, a)}$ and Beat Ruhstaller ${ }^{1,2, b)}$ \\ ${ }^{1}$ Institute of Computational Physics, Zurich University of Applied Sciences, Wildbachstr. 21, 8401 Winterthur, \\ Switzerland \\ ${ }^{2}$ Fluxim AG, Technoparkstrasse 2, 8406 Winterthur, Switzerland
}

(Received 8 January 2015; accepted 25 March 2015; published online xx xx xxxx)

\begin{abstract}
In admittance spectroscopy of organic semiconductor devices, negative capacitance values arise at low frequency and high voltages. This study aims at explaining the influence of self-heating on the frequency-dependent capacitance and demonstrates its impact on steady-state and dynamic experiments. Therefore, a one dimensional numerical drift-diffusion model extended by the heat equation is presented. We calculate the admittance with two approaches: a Fourier method that is applied to time domain data and a numerically efficient sinusoidal steady state analysis $\left(\mathrm{S}^{3} \mathrm{~A}\right)$, which is based on the linearization of the equations around the operating point. The simulation results coincide well with the experimental findings from reference [H. Okumoto and T. Tsutsui, Appl. Phys. Express 7, 061601 (2014)] where the negative capacitance effect of an organic device becomes weaker with better cooling of the structure. Linking the frequency- and time-domain with the Fourier approach supports an effortless interpretation of the negative capacitance. Namely, we find that negative capacitance originates from self-heating induced current enhancement. (C) 2015 AIP Publishing LLC. [http://dx.doi.org/10.1063/1.4916981]
\end{abstract}

\section{INTRODUCTION}

The influence of self-heating on the device performance has been recognized as a major issue in large-area OLEDs. ${ }^{1,2}$ Different remedies were taken to reduce the heat generation on the anode caused by the resistive material. For example, metal grids were added on top of the anode to decrease the potential drop leading to more homogeneous potential, current, temperature, and light distributions. ${ }^{3}$ However, in small organic devices heat generation has only recently been of concern. ${ }^{4}$

In order to characterize an organic semiconductor device, a number of different techniques are available and commonly performed. In admittance spectroscopy, negative capacitance values are often observed at high bias and low frequency and have started a controversial debate. Negative capacitance measurements are well known from Si diodes. Numerous explanations ${ }^{5}$ (and references therein) are named as origin or the negative capacitance is considered a parasitic measurement effect. ${ }^{6}$ First occurrence of negative capacitance in organic LEDs dates back about a decade. ${ }^{7,8}$ In general, the same structure also exhibits positive capacitance at different measurement conditions such as frequency, applied voltage, or temperature. A wide range of origins for the negative capacitance have been brought forward for organic semiconductor devices. $^{9-13}$

Most of the references investigating negative capacitance are limited to bipolar devices and do not analyze the occurrence in hole-only devices (HODs) or electron-only devices (EODs). Little work has been dedicated to unipolar devices so far. For instance, Refs. 14 and 15 report the

${ }^{a)}$ Electronic mail: evelyne.knapp@zhaw.ch

${ }^{b)}$ Electronic mail: beat.ruhstaller@zhaw.ch fabrication and measurement of HOD and EOD exhibiting 50 negative capacitance. The authors attribute the observed neg- 51 ative capacitance effects to interfacial states. Only recently 52 self-heating has been identified as another origin for the neg- 53 ative capacitance in organic semiconductor devices by 54 Okumoto and Tsutsui ${ }^{16}$ for a HOD and bipolar device. Self- 55 heating of the device changes the capacitance value of the 56 structure, even in the case of a small device. In Ref. 16, adding a copper block on top of the HOD led to a reduced negative capacitance value indicating that the device temperature plays an important role.

In this paper, we demonstrate the influence of selfheating in the organic semiconductor with the aid of an extended one dimensional numerical drift-diffusion model on the steady-state current-voltage curve, dark injection transient currents experiment, or also called transient spacecharge limited current (T-SCLC) and, most importantly, on the admittance spectroscopy. We will shed light on the occurrence of the negative capacitance in single carrier devices. Furthermore, we attempt to establish a link between the time and the frequency domain to ease the interpretation of the negative capacitance. Therefore, we simulate in the frequency domain a negative capacitance and check in the time domain if we can find indications leading to a negative capacitance value.

\section{METHODS}

\section{A. Mathematical model}

To model the electrical part of a hole-only device, we apply a one dimensional drift-diffusion model ${ }^{17}$ that includes the Poisson equation (1) for the hole density $p$ and the potential $\psi$ 


$$
\begin{gathered}
\nabla \cdot\left(\epsilon_{0} \epsilon_{r} \nabla \psi\right)=q(-p), \\
J_{p}=-q p \mu \nabla \psi-q D \nabla p, \\
\nabla \cdot J_{p}+q \frac{\partial p}{\partial t}=0 .
\end{gathered}
$$

81 The vacuum permittivity is denoted by $\epsilon_{0}$, the relative per82 mittivity by $\epsilon_{r}$, and the elementary charge by $q$. Furthermore, the continuity equation (3) with the current density $J_{p}(2)$ is solved where the mobility of the organic semiconductor is given by $\mu$.

For simplicity, we assume fixed charge carrier densities at the anode (4) and cathode (5). For a device with thickness $L$ and with $N_{0}$ the number of sites, we write

$$
\begin{gathered}
p(0)=N_{0}, \\
p(L)=N_{0} \exp \left(-\frac{q V_{b i}}{k T}\right),
\end{gathered}
$$

89 where the built-in voltage is denoted by $V_{b i}$.

The classical drift-diffusion model (1)-(3) is only solved in the organic material layer of the hole-only device as shown in Fig. 1.

The electrical model is extended by the heat equation (6), where the temperature is given by $T$. We assume that heat transfer in the device takes place by thermal conduction and that thermal exchange with the surrounding is based on convection and thermal radiation. The heat transfer is calculated for the entire domain of the hole-only device. The heat source term $J_{p}^{2} /(q \mu p)$ is restricted to the organic semiconductor layer. The heat source therein is given by Joule heating, ${ }^{18}$ which is proportional to the electrical resistance of the material. In the other layers, there is no heat source, only heat conduction. In the continuity equation for heat conduction

$$
c \rho \frac{\partial T}{\partial t}=\nabla \cdot(k \nabla T)+\frac{J_{p}^{2}}{q \mu p},
$$

the parameter $c$ stands for the specific heat capacity, $\rho$ for the density, and $k$ for the thermal conductivity.

For the thermal part, we use convective and radiative boundary conditions. The heat flux density $F$ is calculated

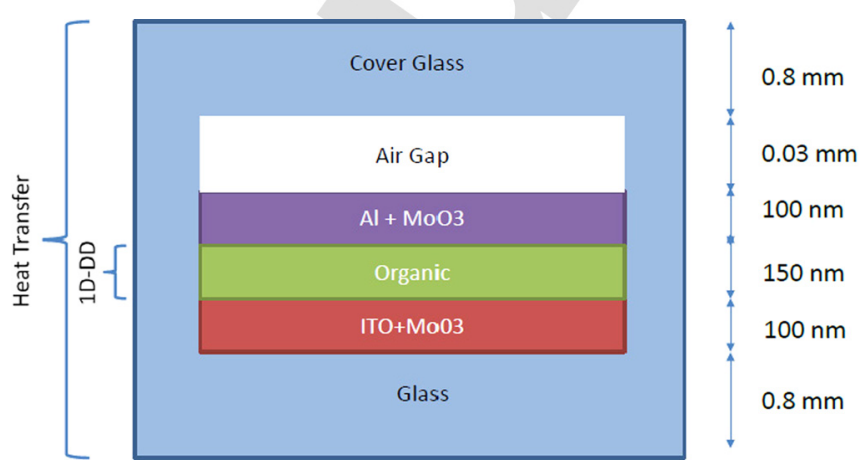

FIG. 1. Structure of HOD and thickness of layers. The heat equations are solved on the entire device domain, while the drift-diffusion model is only solved in the organic layer. from the heat transfer coefficient $h$ and the ambient tempera- 109 ture $T_{r e f}$, further the emissivity is given by $\epsilon$ and the Stefan- 110 Boltzmann constant by $\sigma$

$$
F=-k \nabla T=-h\left(T-T_{r e f}\right)-\epsilon \sigma\left(T^{4}-T_{r e f}^{4}\right)
$$

We assume the same temperature for the convective air flow 112 as for the ambient.

Note that we assume a temperature independent mobil- 114 ity $\mu$, whereas the diffusion $D$ increases with an enhanced 115 temperature since $D$ is proportional to the temperature $T \quad 116$

$$
D=\frac{k T}{q} \mu
$$

We are aware that organic semiconductors in general exhibit 117 a temperature dependent mobility but as we show here, that 118 does not have to be employed to explain negative capaci- 119 tance. In the remainder of this paper, we will denote the one 120 dimensional drift-diffusion model (1 to 3 ) with 1D-DD and 121 the extended model (1 to 3 and 6 ) with 1D-EDD.

\section{B. $S^{3} A$ and Fourier simulation method}

In this section, we introduce two different approaches 124 for calculating the admittance of a sample device. For the 125 sample device with all parameters given in Table I, we only 126 consider the organic semiconductor layer at a constant de- 127 vice temperature of $300 \mathrm{~K}$ and solve the 1D-DD model. At a 128 later stage, we also apply the methods to the 1D-EDD 129 model.

The admittance $Y=\frac{I^{a c}}{V^{a c}}$ requires the determination of the 131 ac current response $I^{a c}$ to a harmonic voltage modulation $V^{a c}{ }_{132}$ $=V_{\text {offset }}+V_{0} \cos (\omega t)$. The frequency-dependent admittance 133

TABLE I. Material parameters for all simulations.

\begin{tabular}{lccc}
\hline \hline Thermal $^{25}$ & & & \\
\hline Material & $\begin{array}{c}\text { Density } \\
{\left[\mathrm{kg} / \mathrm{m}^{3}\right]}\end{array}$ & $\begin{array}{c}\text { Heat capacity } \\
{[\mathrm{J} /(\mathrm{kgK})]}\end{array}$ & $\begin{array}{c}\text { Conductivity } \\
{[\mathrm{W} /(\mathrm{Km})]}\end{array}$ \\
\hline Glass & $2.6 \times 10^{3}$ & $8.2 \times 10^{2}$ & 3.0 \\
ITO & $7.2 \times 10^{3}$ & $3.4 \times 10^{2}$ & 8.0 \\
Organic & $1.2 \times 10^{3}$ & $1.7 \times 10^{3}$ & $2.0 \times 10^{-1}$ \\
Al & $3.9 \times 10^{3}$ & $9.0 \times 10^{2}$ & $2.0 \times 10^{1}$ \\
Air gap & 1.2 & $1.0 \times 10^{3}$ & $2.5 \times 10^{-2}$
\end{tabular}

Electrical

Material Mobility $\left[\mathrm{m}^{2} /(\mathrm{Vs})\right] \quad$ Site density $\left[\mathrm{m}^{-3}\right] \quad$ Relative permittivity

\begin{tabular}{lll}
\hline Organic & $5 \times 10^{-10}$ & $1 \times 10^{26}$
\end{tabular}

Boundary conditions

\begin{tabular}{lccc}
\hline B.C & Parameter & Value & Units \\
\hline Thermal & $\mathrm{h}$ & $10 / 50$ & $\mathrm{~W} /\left(\mathrm{m}^{2} \mathrm{~K}\right)$ \\
& $\epsilon$ & 0.92 & \\
Electrical & $V_{b i}$ & 0.6 & $\mathrm{~V}$ \\
\hline
\end{tabular}


can then by decomposed into the conductance $G$ and capacitance $C$ according to $Y(\omega)=G(\omega)+i \omega C(\omega)$.

The first approach is the $\mathrm{S}^{3} \mathrm{~A}$. In Ref. 22, a more detailed description of the method is given and was used previously. $^{22-24}$ We first solve the steady-state equations for $V_{\text {offset }}$ and linearize the system of equations around this operating point to get the ac equations. For each frequency $\omega$, we solve the linear ac equations and calculate thereof the admittance $Y(\omega)$.

In the second approach, the transient current response $I(t)$ to a small voltage step $\Delta V$ is monitored and Fourier transformed. This leads to the following expression for the capacitance $C$ and conductance $G$ as elaborated in detail in Ref. 5:

$$
\begin{aligned}
& C(\omega)=C_{\text {geom }}+\frac{1}{\Delta V} \int_{0}^{\infty} \delta j(t) \cos (\omega t) d t, \\
& G(\omega)=G(0)+\frac{\omega}{\Delta V} \int_{0}^{\infty} \delta j(t) \sin (\omega t) d t .
\end{aligned}
$$

The transient current density $\delta j(t)$ is defined as

$$
\delta j(t)=I(t)-I(\infty),
$$

so that $\delta j(t) \rightarrow 0$ in the steady-state limit $(t \rightarrow \infty)$.

Applying the formula for the capacitance (9) and conductance (10) to the transient current response to a small voltage step as shown in Fig. 2, we can calculate the capacitance $C$ and conductance $G$ of the sample device. The transient current response in Fig. 2 shows according to Ref. 21 a cusp at

$$
t_{D I}=0.786 \frac{L^{2}}{\mu\left(V-V_{b i}\right)} .
$$

This formula can be useful for mobility determination.

A comparison between the two methods is displayed in Fig. 3. In the work of Ref. 19 , the value of $0.75 C_{\text {geom }}$ is obtained below the transit frequency. Note that capacitance in our case is slightly enhanced due to diffusion which was neglected in Ref. 19 , but we have previously shown ${ }^{20}$ that the low-frequency capacitance reaches values of $0.8 C_{\text {geom }}$ (not simply $0.75 C_{\text {geom }}$ ) because the numerical model considers

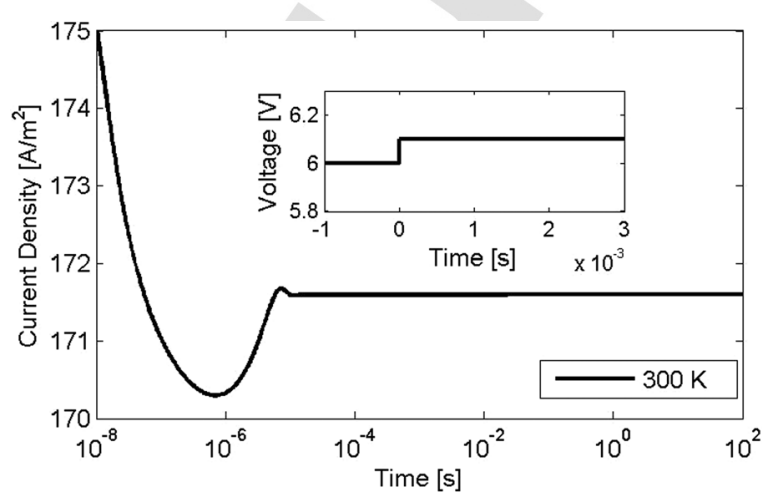

FIG. 2. The current density response over time is shown. A cusp is found and eventually the steady-state is reached. In the inset, the small voltage step is displayed.

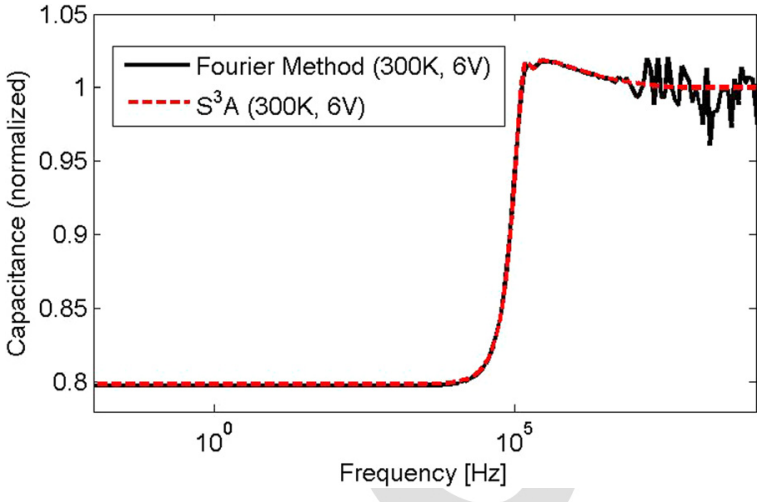

FIG. 3. The capacitance is calculated with two approaches: the black line represents the $S^{3} A$ solution, while red stands for the Fourier method. At high frequency, the accuracy of the Fourier method is clearly limited.

charge diffusion, too. The negative differential susceptance 164 $-\Delta B=-\omega\left(C(\omega)-C_{\text {geom }}\right)$ yields a maximum at $f_{\text {max }}$ due to 165 the transit time effect leading to

$$
\mu=1.85 \frac{L^{2} f_{\max }}{\left(V-V_{b i}\right)} .
$$

Comparing the two methods shows that the Fourier 167 method is less accurate at high frequencies as the accuracy is 168 dependent on the size of the time step $\Delta t$ in the transient cur- 169 rent response $\left(\Delta t \ll 2 \pi f_{h i g h}^{-1}\right)$. Moreover, the applied voltage 170 step $\Delta V$ must be small in order to be in the linear regime of 171 the device, yet big enough to get an accurate numerical cur- 172 rent resolution.

Despite the fact that the $S^{3} A$ provides superior numeri- 174 cal accuracy, the Fourier method allows for an effortless 175 interpretation of the negative capacitance effects in the time- 176 domain. We have now two methods at hand that allow for 177 the determination of the capacitance $C$ and conductance $G .178$ The two methods will be applied in Sec. IV.

\section{STATIONARY SIMULATIONS}

First of all, we turn to steady-state simulations and com- 181 pare the results of the 1D-DD and 1D-EDD model. All pa- 182 rameters are given in Table I.

183

In a first step, we perform the most common characteri- 184 zation technique and calculate the current-voltage curve for 185 the 1D-DD model for a constant device temperature of 186 $300 \mathrm{~K}$. This result is shown as the black line in Fig. 4. In a 187 next step, we extend the model by the heat equation on the 188 entire domain and recalculate the current-voltage curve 189 (allowing for self-heating of the device) and obtain the red 190 curve (1D-EDD). At high voltage, self-heating clearly 191 enhances the current. A change in temperature enhances dif- 192 fusion (see Eq. (8)) and leads to more carriers in the device. 193 Thus, the current increases.

In Fig. 5, the temperature profile of the entire device at 195 $6 \mathrm{~V}$ is displayed at steady-state. We notice that the air gap is 196 mainly responsible for the temperature difference in the de- 197 vice. The inset shows the temperature distribution of the 198 electrodes and the organic layer as indicated by the arrow in 199 


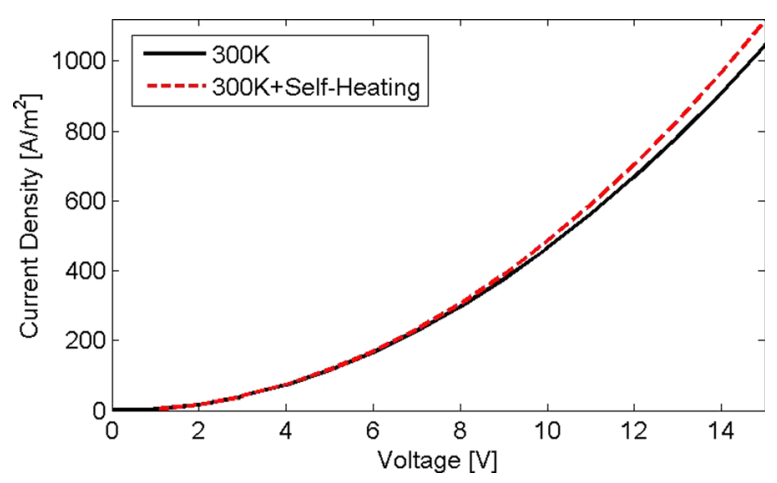

FIG. 4. Current-voltage curves for constant temperature in the device (black) and extended model with self-heating (red). Self-heating rises the current at high voltage.

Fig. 5. Joule heating leads to the enhanced temperature in the organic layer.

\section{TRANSIENT SIMULATIONS}

The difference between the two models becomes even more distinct when we look at time-dependent measurements and simulations. Calculating the capacitance for the 1D-DD model leads to the well known curve for a HOD as shown in Fig. 3 or Refs. $22-24$. The capacitance step at around $10^{5} \mathrm{~Hz}$ can be related to the charge carrier mobility. ${ }^{22}$ Namely, at higher frequency the capacitance approaches the geometric capacitance of the device. Solving the 1D-EDD model with heat transport changes the result vastly. At low frequency and high bias, negative capacitance values are found as shown in Fig. 6. The capacitance step remains at the same position for the same applied voltage independent of selfheating. In Fig. 6, the capacitance values for different applied voltages are displayed for the 1D-EDD model. The higher the applied bias, the more pronounced is the negative capacitance effect.

In order to compare the effect of cooling in our model with measurements in Ref. 16, we calculate the capacitance of a HOD with thickness $150 \mathrm{~nm}$ as displayed in Fig. 7. By changing the value for the convective cooling parameter $h$

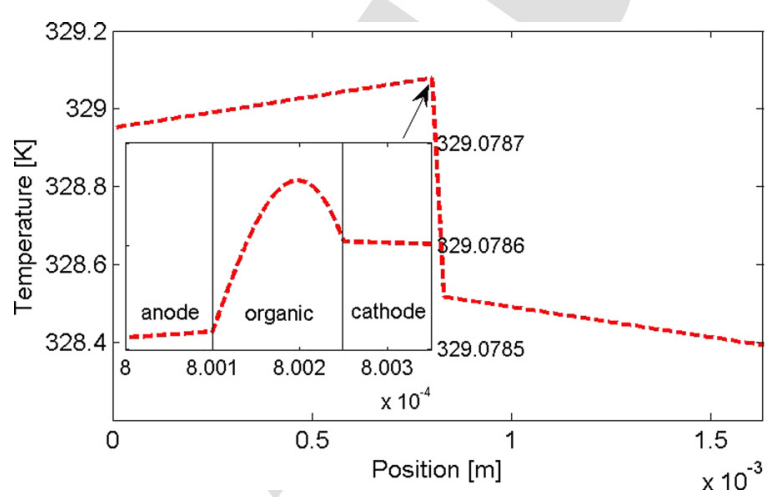

FIG. 5. For an operating voltage of $6 \mathrm{~V}$, the temperature distribution over the entire device is shown in steady state. The biggest temperature drop is over the air gap between the cathode and the encapsulation. The arrow indicates the electrodes and organic layer. They are zoomed in the inset. Joule heating acts as a heat source in the organic material. The generated heat is then transferred to the electrodes.

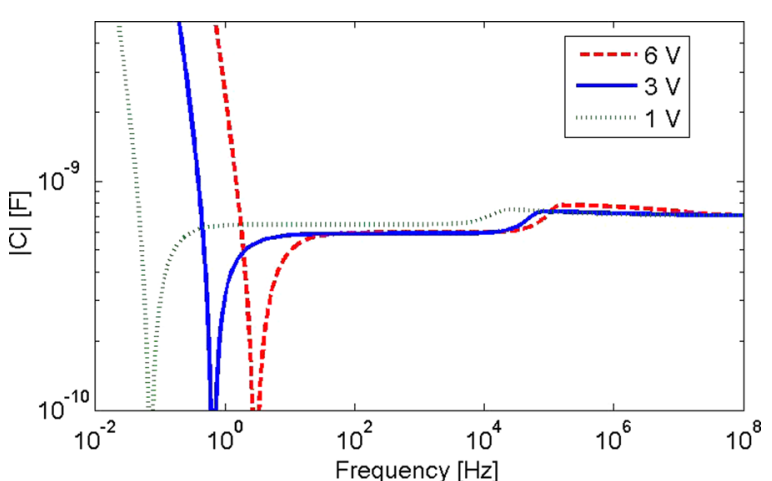

FIG. 6. The absolute value of the capacitance of the 1D-EDD model for different voltages. Including the heat equation leads to a considerable change in the capacitance at low voltage. At low bias, the effect is decreasing.

(see 7), we can simulate the effect of a copper block and get 223 an excellent agreement with measurement as shown in Fig. 4224 of Ref. 16. Cooling makes the device less inductive. The 225 thermal parameters for the simulation are taken from Ref. 226 25.

The simulations can capture the features of the measure- 228 ments. Without the copper block, the capacitance becomes 229 increasingly negative with lower frequency and only flattens 230 at around $10^{-3} \mathrm{~Hz}$. Cooling the device flattens the capaci- 231 tance curves at around $10^{-2} \mathrm{~Hz}$. The two situations show 232 that self-heating can lead to a negative capacitance, even in a 233 hole-only device. To shed light on the source of the negative 234 capacitance, we calculate the transient response for the 1D- 235 DD and 1D-EDD model. In terms of physics, this implies 236 that in the first case the device temperature remains constant 237 and in the second case self-heating is included. In Fig. 8, the 238 transient current density responses are shown for a voltage 239 step of $0.1 \mathrm{~V}$. The current density values of the top curve are 240 higher due to self-heating. Both curves show a transit-time 241 cusp at $t_{D I}$ regardless of self-heating. This is in agreement 242 with the top curve which, however, still increases after sev- 243 eral orders of magnitude in time after the cusp. The heat gen- 244 erated in the organic layer is conducted through the device 245 increasing the overall temperature, which again leads to a 246 higher current density in the organic layer. This is a slow 247 thermal process and the final steady-state current density 248

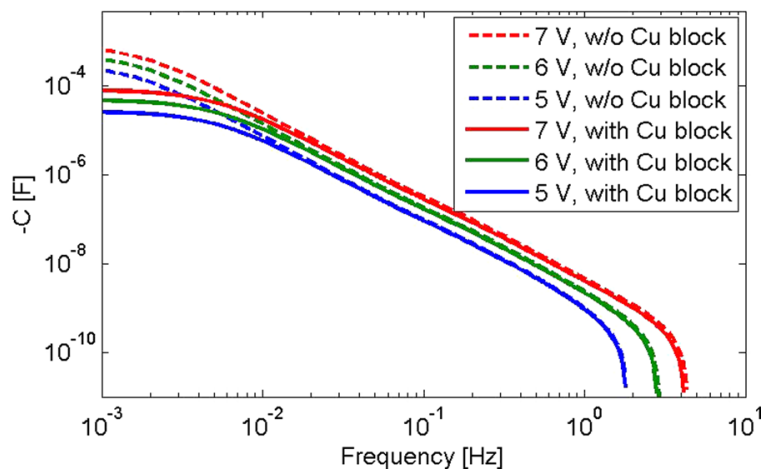

FIG. 7. Simulation of measurement features for different voltages and with and without the copper block. The cooling is modeled by changing the convective cooling boundary conditions. Cooling leads to smaller absolute capacitance values. 


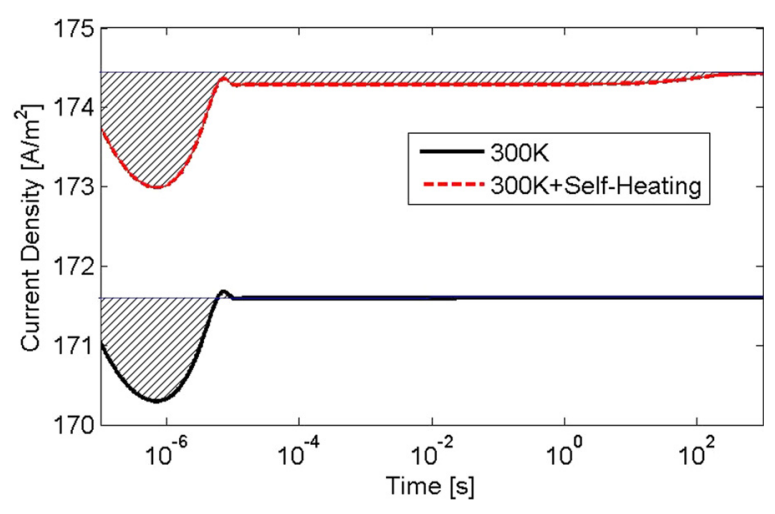

FIG. 8. Current density response for the 1D-DD and the extended 1D-EDD model to a voltage step of $0.1 \mathrm{~V}$ at an operating voltage of $6 \mathrm{~V}$. The blue line represents the steady-state value and the reference level for the integral in Eq. (14) for the two cases. In the case of self-heating, the shaded area under the reference line is significantly bigger resulting in a negative capacitance value.

value is only reached after a while, approx. $10^{2} \mathrm{~s}$ here. Transforming the data from the time domain to the frequency domain according to Eq. (9) reproduces the negative capacitance effect nicely for the device with self-heating. The absolute value of the capacitance is shown in Fig. 6 for different voltages. The curve corresponding to Fig. 8 is at $6 \mathrm{~V}$, while the curve without self-heating corresponds to Fig. 3. To understand the negative capacitance occurrence, Ershov ${ }^{5}$ has pointed out that for low frequencies formula (9) can be approximated by

$$
C(0)=C_{\text {geom }}+\frac{1}{\Delta V} \int_{0}^{\infty} \delta j(t) d t .
$$

This implies that the integral that we add to the geometrical capacitance $C_{\text {geom }}$ only depends on the transient current response with respect to its steady-state value as indicated with the blue line. The shaded area for the 1D-DD is mainly under the blue line leading to a negative contribution. It is responsible for the reduction of the geometric capacitance to a value of approximately $0.8 C_{\text {geom }}$ in Fig. 3 . The shaded area under the blue line is clearly bigger in the case of selfheating leading to an even more negative capacitance contribution such that expression (14) turns negative. Note that we used a logarithmic time scale in Fig. 8 and the initial contribution to the dark injection transient cusp is relatively small in comparison to the rest of the shaded area. With this approach, we can easily classify a device with respect to negative capacitance from its transient current density response. The temperature increase due to self-heating enhances the current density order of magnitudes later in time, which is thus responsible for the negative capacitance effect.

In order to investigate the resulting time scale for heating, in Fig. 9 the simulated transient current density response is simulated for two distinct thermal model assumptions. The case of a thermal model including the entire device domain with the glass substrate is compared to a case where a reduced thermal model domain only including the organic semiconductor layer is considered. If the entire device including the glass substrate is included in the thermal model

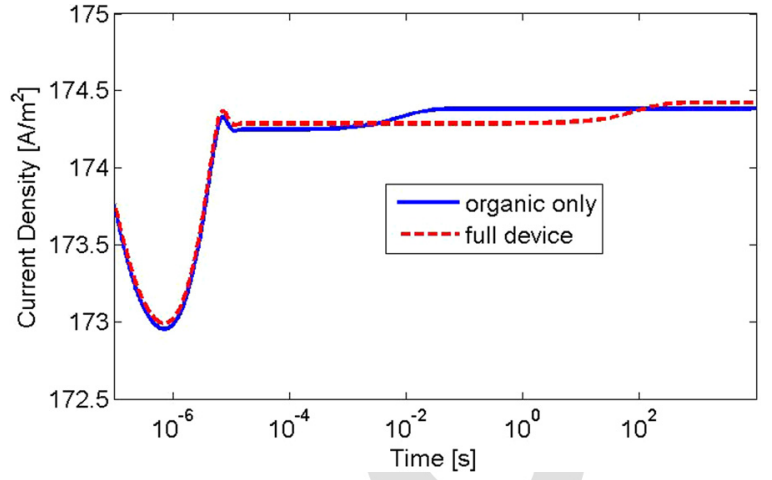

FIG. 9. The simulated transient response to a voltage step of $0.1 \mathrm{~V}$ at an operating voltage of $6 \mathrm{~V}$ is shown for two assumptions of the thermal modeling domain. If the modeling domain only contains the organic semiconductor layer then the self-heating induced current rise occurs within milliseconds. However, if the entire device including the glass substrate is included in the thermal model domain, then the characteristic time scale for the self-heating-induced current rise occurs at approx. $100 \mathrm{~s}$ after turn on, which is a realistic warm up period often observed in experiment. The time of the dark injection transient cusp is determined by the drift-diffusion model and thus identical for both assumptions of the thermal model domain.

domain, then the characteristic time scale for the self-heat- 285 ing-induced current rise is approx. $100 \mathrm{~s}$ after turn on. The 286 size of the simulation domain changes the observed time lag 287 when the self-heating starts. The larger the simulation do- 288 main, the longer it takes to heat up and the hotter the device 289 becomes. This is in agreement with the characteristic time 290 $t_{\text {char }}$

$$
t_{c h a r}=\frac{L_{t o t}^{2}}{k_{e} /\left(c_{e} \rho_{e}\right)},
$$

for the heat equation. The total device thickness of all layers 292 and glass is denoted by $L_{t o t}$ and the effective thermal materi- 293 als parameters have a subscript $e$. As expected, the dark 294 injection transient time cusp remains at the same position for 295 the two devices. Comparing the capacitance of the two devi- 296 ces leads thus to a different frequency where the negative ca- 297 pacitance effects set in as shown in Fig. 10. The 298 encapsulation of an organic device is thus crucial for its elec- 299 trical performance and can change the negative capacitance 300

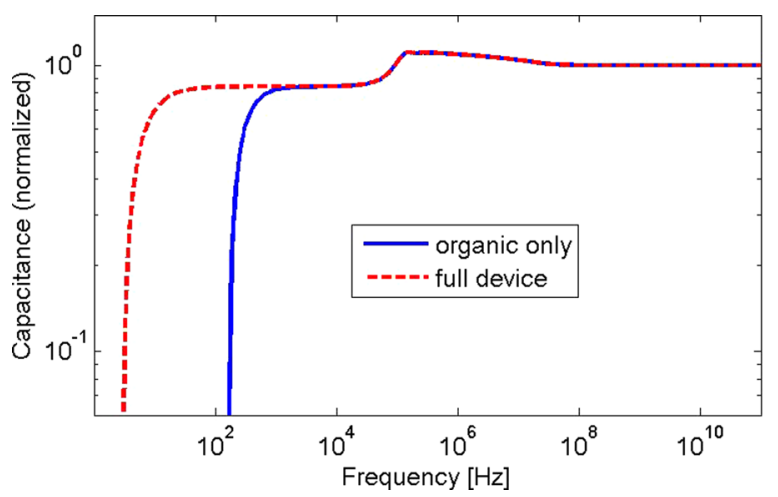

FIG. 10. The corresponding frequency-dependent capacitances to the transients in Fig. 9 are shown. For the more realistic thermal modeling domain including the glass substrate, the drop in capacitance (leading eventually to negative values) occurs at smaller frequencies, then in the simplified thermal model where only the organic semiconductor is considered. 
behavior. Depending on the measurement regime, some scientists may claim that negative capacitance does not even occur, but changing the frequency range, the total device thickness or material around the organic semiconductor may reveal a negative capacitance effect. As indicated in Eq. (15), the characteristic time depends on the total thickness of all layers $L_{t o t}$ and on the thermal properties of all layers involved.

\section{CONCLUSIONS}

To identify and understand the origin of negative capacitance in organic semiconductor devices, the 1D-DD model of the organic semiconductor layer has been extended by the heat equation on the entire domain. With this model that includes Joule heating as heat source in the semiconductor layer, negative capacitance effects in single carrier devices were investigated. As opposed to large-area devices where the heat generation of the electrodes is considered, we focus on the self-heating in the organic semiconductor layer.

The model demonstrates the effect of self-heating in terms of the current voltage curve, but more importantly and distinctively, in dynamic characterization. We emphasize that our conclusions were drawn from a model in 1D and there is no need to resort to 3D. The model agrees very nicely with measurements from Ref. 16. Previously, other explanations as trap dynamics, interfacial states, recombination, etc., were named as origin of negative capacitance. These effects, however, would not be affected by adding a copper block on top of the device and could not reproduce a negative capacitance effect by simulation. The 1D-DD model extended by heat conduction allows for a comprehensive and consistent description of charge transport taking all major physical processes into account as opposed to simpler models such as presented in Ref. 16 or equivalent circuit models. $^{14,15}$

With the aid of the Fourier method, an accessible explanation of the negative capacitance has been presented which allows for an interpretation in the time domain, which is generally more intuitive than the frequency domain.

The analysis, however, has been restricted to a trap-free unipolar, single layer sample with constant mobility and simplified boundary conditions. As a next step, a quantitative comparison between measurement and simulation is desired. The model can be further improved by including a temperature-dependent mobility model. In unipolar samples, the negative capacitance effect might be overshadowed by adding trap states if they act on a similar time scale. Trap states usually enhance the capacitance at low frequency, ${ }^{22}$ while self-heating lowers the capacitance. The signature of trap states in the current voltage curve would lead to a reduction of the current density. In the DITC, a decay after the transient-time cusp represents the trapping process.
The simulations show the importance of self-heating in 352 small devices and confirm self-heating as origin of negative 353 capacitance. Depending on the structure of the samples, this 354 might lead to undesired effects in the performance. Heat dis- 355 sipation should be taken into account when fabricating or- 356 ganic semiconductor devices and be considered in any 357 electrical characterization technique regardless of $\mathrm{dc}$, ac, or 358 transient.

In conclusion, it is crucial to model the heat generation 360 and transport in organic semiconductor devices in order to 361 obtain accurate simulation results at typical operating condi- 362 tions, i.e., current densities of organic semiconductor devi- 363 ces. So far, the role of self-heating, especially in small 364 devices, has been underestimated. It is very likely that our 365 findings are not restricted to organic semiconductor devices 366 but would hold for wider classes of materials and devices. $\quad 367$

\section{ACKNOWLEDGMENTS}

E. Knapp acknowledges gratefully financial support from 370 the Swiss National Science Foundation through a Marie- 371 Heim-Vögtlin Fellowship. The authors would like to thank 372 Martin Neukom from Fluxim AG for fruitful discussions. $\quad 373$

${ }^{1}$ M. Slawinski, D. Bertram, M. Heuken, H. Kalisch, and A. Vescan, Org. 375 Electron. 12, 1399-1405 (2011). 376

${ }^{2}$ J. W. Park, D. C. Shin, and S. H. Park, Semicond. Sci. Technol. 26, 377 034002 (2011). 378

${ }^{3}$ S. Harkema, S. Mennema, M. Barink, H. Rooms, J. S. Wilson et al., Proc. 379 SPIE 7415, 74150T (2009).

${ }^{4}$ A. Fischer et al., Adv. Funct. Mater. 24(22), 3367-3374 (2014). 381

${ }^{5}$ M. Ershov, H. C. Liu, L. Li, M. Buchanan, Z. R. Wasilewski, and A. K. 382 Jonscher, IEEE Trans. Electron. Devices 45(10), 2196 (1998). 383

${ }^{6}$ K. S. A. Butcher, T. L. Tansley, and D. Alexiev, Solid-State Electron. 384 39(3), 333-336 (1996).

${ }^{7}$ H. C. F. Martens, J. N. Huiberts, and P. W. M. Blom, Appl. Phys. Lett. 77, 386 $1852(2000)$

${ }^{8}$ H. L. Kwok, Solid-State Electron. 47, 1089 (2003).

${ }^{9}$ I. N. Hulea, R. F. J. van der Scheer, H. B. Brom, B. M. W. Langeveld- 389 Voss, A. van Dijken, and K. Brunner, Appl. Phys. Lett. 83, 1246 (2003). 390

${ }^{10}$ L. S. C. Pingree et al., Appl. Phys. Lett. 86, 073509 (2005). 391

${ }^{11}$ H. H. P. Gommans, M. Kemerink, and R. A. J. Janssen, Phys. Rev. B 72, 392 235204 (2005).

${ }^{12}$ E. Ehrenfreud et al., Appl. Phys. Lett. 91, 012112 (2007).

${ }^{13}$ L. Nuo et al., Chin. Phys. B 20(2), 027306 (2011).

${ }^{14}$ G. Garcia-Belmonte et al., Chem. Phys. Lett. 455, 242 (2008).

${ }^{15}$ G. Garcia-Belmonte et al., Synth. Met. (2008).

${ }^{16}$ H. Okumoto and T. Tsutsui, Appl. Phys. Express 7, 061601 (2014).

398 J. Appl. Phys. 108, 054504-0545012 (2010).

${ }^{18}$ J. Piprek, Semiconductor Optoelectronic Devices: Introduction to Physics 401 and Simulation (Academic Press, 2013).

${ }^{19}$ J. Shao and G. T. Wright, Solid-State Electron. 3, 291 (1961).

${ }^{20}$ Optoelectronic Devices and Properties, edited by O. Sergiyenko ( 2011).

${ }^{21}$ A. Many and G. Rakavy, Phys. Rev. 126, 1980 (1962).

${ }^{22}$ E. Knapp and B. Ruhstaller, Appl. Phys. Lett. 99, 093304 (2011).

${ }^{23}$ E. Knapp and B. Ruhstaller, J. Appl. Phys. 112, 024519 (2012).

${ }^{24}$ E. Knapp and B. Ruhstaller, Opt. Quantum Electron. 42, 667-677 (2011).

${ }^{25}$ X. Qi and S. R. Forrest, J. Appl. Phys. 110, 124516 (2011). 